\title{
ON A PROBLEM IN CLOSURE*
}

\author{
BY VIRGIL SNYDER
}

The following note concerns finite groups of birational transformations which leave an algebraic curve of genus 1 invariant.

Let the curve be expressed as a $C_{4}$ in $S_{3}$, intersection of two general quadric surfaces. This curve is invariant under the linear group $G_{8}$, of order eight, generated by the harmonic homologies defined by the self-conjugate tetrahedron associated with $C_{4}$. The points of $C_{4}$ are thus arranged in sets of 8 , forming a linear $I_{8}{ }^{1}$ of genus 0 . If the curve be projected upon a plane from an arbitrary point, a plane quartic $C_{4}$ results with nodes at $K_{1}, K_{2}$. The four central homologies become four perspective quadratic involutions $T_{i}$ with centers $O_{i}$ not on $C_{4}$; the three axial involutions become three non-perspective quadratic inversions, with fundamental points $O_{i k}$ for $T_{i} T_{k}=$ $T_{k} T_{i}=T_{k i}=T_{l m}$ at the diagonal points of the quadrangle $\mathrm{O}_{1} \mathrm{O}_{2} \mathrm{O}_{3} \mathrm{O}_{4}$. The nodes $K_{1}, K_{2}$ are the other fundamental points for all seven operations. $\dagger$

From the theorem of Bertini it follows that in any plane nodal quartic one and only one conic can be found meeting

* Presented to the Society, September 8, 1926.

$\dagger$ A brief synthetic outline of the properties of $G_{8}$ was first given by C. Segre, Su una trasformazione irrazionale dello spazio . . . , Giornale di Matematiche, vol. 21 (1883), pp. 355-378. This was amplified in connection with a larger problem by D. Montesano, Su alcuni gruppi chiusi di trasformazioni involutorie nel piano e nello spazio, Atti Istituto Veneto, (6), vol. 6 (1888), pp. 1425-1444. It is also contained in the papers by $\mathrm{K}$. Meister, Ueber die Systeme, welche durch Kegelschnitte mit einem gemeinsamen Polardreieck, bez. durch Flächen zweiten Grades mit einem gemeinsamen Polartetraeder gebildet werden, Zeitschrift der Mathematik und Physik, vol. 31 (1886), pp. 321-347; vol. 34 (1889), pp. 6-24; 73-91 and by H. E. Timerding, Ueber die quadratische Transformation durch welche die Ebenen des Raumes in ein System von Flächen zweiter Ordnung mit gemeinsamen Poltetraeder ïbergefïhrt werden, Annali di Matematica, (3), vol. 1 (1898), pp. 95-117. 
any line through the node in two points which form with the residual intersections of the line and the quartic a harmonic set. Using this conic as conic of invariant points and the node as center in a perspective quadratic inversion, the quartic is transformed into itself. In case there are two nodes there is a conic of Bertini associated with each, and the conic associated with one node passes through the other. Let these operations be denoted by $S_{1}, S_{2}$. In a recent memoir* the question is raised whether the group generated by $S_{1}, S_{2}$ and $G_{8}$ can be finite, but the author does not answer it. The answer may be difficult if the method is restricted to pure geometry. By assistance of the parametric representation in terms of elliptic functions it can be answered in every case.

Let

$$
F=x_{1}^{2}-x_{2}^{2}+x_{3}^{2}-x_{4}^{2}=0, \quad \sum a_{i} x_{i}^{2}=0
$$

define the curve. The tetrahedron of reference is the selfconjugate tetrahedron of the pencil of quadrics, and the equations of the harmonic homology $H_{1}$ with center at vertex $(1,0,0,0)$, invariant plane $x_{1}=0$, are $p x_{1}^{\prime}=-x_{1}, p x_{i}{ }^{\prime}=x_{i}$, $i=2,3,4$; similarly for $H_{2}, H_{3}, H_{4}$.

By projecting $F=0$ upon $x_{4}=0$ from $(1,1,1,1)$, the operation $H_{1}$ becomes the perspective inversion

$$
\left\{\begin{array}{l}
x_{1}^{\prime}=\left(x_{2}-x_{3}\right)\left(x_{1}-x_{2}-x_{3}\right), \\
x_{2}^{\prime}=x_{2}\left(x_{1}-x_{2}+x_{3}\right), \\
x_{8}^{\prime}=x_{3}\left(x_{1}+x_{2}-x_{3}\right) .
\end{array}\right.
$$

The center is $O_{1}=(1,0,0)$, and the other fundamental points are

$$
K_{1}=(0,1,1), \quad K_{2}=(1,1,0) .
$$

The conic of invariant points is

$$
\left(x_{1}-x_{2}\right)^{2}+2 x_{1} x_{3}-x_{3}^{2}=0 .
$$

* E. Ciani, Le quartiche piane invertibili, Giornale di Matematiche, vol. 57 (1919), 47 pages. 
Similar expressions exist for $T_{i}, i=2,3,4$, and for $T_{i k}, O_{4}=$ $(1,1,1)$. The triangle formed by any three of the points $O_{i} O_{k} O_{l}$ is self-conjugate as to the conic of invariant points associated with the fourth.*

Among the space quartics belonging to the same selfconjugate tetrahedron are $\infty^{1}$ through the center of projection. This pencil is projected into a pencil of plane cubics through $K_{1}, K_{2}$, the four vertices $O_{1}, O_{2}, O_{3}, O_{4}$, and the three diagonal points $O_{i k}$. Hence by suppressing any side of the quadrangle, the four remaining basis points and $K_{1}, K_{2}$ lie on a conic.

The equation can be reduced to the form

$$
k_{1} x_{1}\left(x_{2}-x_{3}\right)\left(x_{2}+x_{3}-x_{1}\right)+k_{2} x_{2}\left(x_{3}-x_{1}\right)\left(x_{1}-x_{2}+x_{3}\right)=0 .
$$

The points $K_{1}, K_{2}$ form a pair of conjugate points in the Geiser net determined by the other seven basis points. The line joining them meets any $C_{3}$ of the pencil in just one point, which with the seven basis points makes a group of $I_{8}$ on that curve. Any position on $C_{3}$ can be chosen for one of them, and then the other is uniquely fixed.

The points $O_{i}$ are all cotangential, and any one of them can be assumed at will. The fundamental points $O_{i k}$ are collinear with $O_{i} O_{k}$ and with $O_{l} O_{m}$. Associated with any point $P$ on $C_{3}$ are the points of tangency of the four tangents from $P$. The other three from the first tangential of $P$ have their points of contact at $O_{i k}$. Expressed in parametric form, assuming the form to which every elliptic cubic can be reduced, that three points are collinear when the sum of their parameters is congruent to zero, then if $P$ has the parameter $u$, we have

$$
\begin{gathered}
O_{1}=-\frac{u}{2}, \quad O_{2}=-\frac{u}{2}+\omega, \quad O_{3}=-\frac{u}{2}+\omega^{\prime}, \\
O_{4}=-\frac{u}{2}+\omega+\omega^{\prime}, \quad O_{12}=u+\omega, \quad O_{13}=u+\omega^{\prime}, \\
O_{14}=u+\omega+\omega^{\prime} .
\end{gathered}
$$

* The equations of the operations of the group and those of an $\infty^{2}$ family of binodal quartics each invariant under it were determined in my seminar by Miss Bertha I. Hart, of Western Maryland College. 
Let $K_{1}$ have the parameter $v$; then $K_{2}$ has the parameter $-u-v$. A conic through $K_{1}$ and through the four points remaining of the seven basis points when three on any line have been suppressed will also pass through $K_{2}$.

The involution in which $K_{1}, K_{2}$ are a pair of conjugate points is that having the polar conic at $P$ for conic of invariant points, and $P$ for vertex.

If $K_{1}, K_{2}$ be regarded as fixed, then a cubic of the pencil is fixed by its residual intersection with $K_{1}, K_{2}$. Since $P_{1} K_{1}$ can be chosen at will and satisfy all the conditions of the problem it follows that the group generated by $K_{1}, O_{1}$ is generally of infinite order. If finite, $K_{1}, G_{8}$ is also finite.

Since $P$ can be chosen at will on $C_{3}$ and $K_{1}$ can be chosen at will, there are $\infty^{2}$ groups of $I_{8}$ in the plane. As groups of operations there appear in sets of pencils, those of any set $\left(O_{i}\right.$ on any curve of the pencil) having the three operations $T_{i k}$ in common, so far as the transformations of points on the curve are concerned.

These results can be interpreted in terms of $C_{4}$ in space directly. By an appropriate linear transformation we may write*

$$
x_{1}=\sigma_{1}(2 u), \quad x_{2}=\sigma_{2}(2 u), \quad x_{3}=\sigma_{3}(2 u), x_{4}=\sigma(2 u),
$$

the $\sigma_{i}$ being those defined in the Schwarz-Weierstrass Formeln und Lehrsätze, pp. 21-2.

Four points with parameters $u_{1}, \cdots, u_{4}$ are collinear if $\Sigma u_{i}=0$. The operations of $G_{8}$ are defined as follows:

$$
\begin{array}{ll}
u^{\prime}=-u+a+\omega^{\prime} & \text { is } H_{1}, \\
u^{\prime}=-u+\omega^{\prime} & \text { is } H_{2}, \\
u^{\prime}=-u+\omega & \text { is } H_{3}, \\
u^{\prime}=-u & \text { is } H_{4},
\end{array}
$$

from which the $H_{i k}$ at once follow.

A quadric of the pencil through $C$ passes through any given point $P$. The quadric contains a generator of each system,

* E. Lange, Die sechzehn Wendeberïhrungspunkte der Raumcurve vierter Ordnung, erster Species, Schlömilchs Zeitschrift, vol. 28 (1883), pp. 1-23. 
through $P$, each meeting $C_{4}$ twice. If this quadric be projected stereographically from $P$, the plane quadric will have a double point at $K_{1}$ on one generator and at $K_{2}$ on the other. Consider the line $P K_{1}$. It contains $P_{1}, P_{2}$ on $C_{4}$ and any plane through $P K_{1}$ meets $C_{4}$ in two points $P_{3}, P_{4}$ which project into two points collinear with $K_{1}$. These points are interchanged by $S_{1}$, hence in space we may choose $u_{1}$ and $u_{2}$ at will, then pass a plane through the points determined by them and any third point $u_{3}$. The space operation consists in interchanging the point $u_{3}$ with the fourth point $u_{4}$ as the plane turns about $u_{1} u_{2}$, as $u_{3}$ describes $C_{4}$.

The operation has the form

$$
u^{\prime}=-u+c,
$$

where $c$ is any given constant. If this be associated with $H_{i}$ the product is not periodic unless $c$ is of the form $r \omega+r^{\prime} \omega^{\prime}$ wherein $r, r^{\prime}$ are both rational. But if this product is rational, then the group generated by $S$ and $G_{8}$ is finite. The operation $S_{2}$ is defined by

$$
u^{\prime}=-u-c,
$$

hence the necessary and sufficient condition that $S_{1}, S_{2}, G_{8}$ generate a finite group is that $S_{1}, S_{2}$ generate a finite group.

Cornell University 